Fixing by horizontal angles is generally to be preferred to fixing by cross bearings, but if this method is not available because available marks are not wellplaced, and a cocked hat results from laying down three position lines obtained from compass bearings, the degree of accuracy of each position line should be estimated and three position sectors drawn. The ship's position should then be estimated to lie at that corner of the star of certainty which will give the safest margin of error.

Many mariners will no doubt argue that drawing stars of certainty on their navigational charts every time a fix is required, is not a practical solution to the problem, and I would tend to agree with them in most cases. However, the above simple treatment serves to emphasize the need for care when setting courses from fixes obtained from three compass bearings. The margin of error allowed should clearly be more than that which would result from assuming the true position of the ship to be at one corner of the cocked hat. Furthermore, the prudent seaman should continue to regard his plotted fixes, if they appear to be perfect, with caution and suspicion.

\title{
Error of the Closest Point of Approach
}

\author{
from N. Sameshima
}

THE problem of errors in radar plotting were considered by Capt. H. Topley (11, 167) and Capt. F. J. Wylie (12, 198), and it was shown that the error in the estimated distance of the C.P.A. depends upon mean range and range change in plotting interval. I want to introduce the term rate of error in the estimated distance of C.P.A., in the same way that Topley shows the percentage error in the estimated speed.

In Fig. $1, O$ is own ship, $A$ is the first plotted point

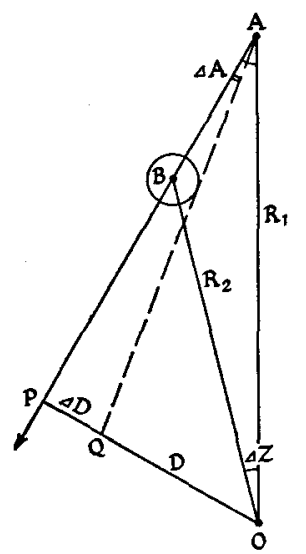

Fig. 1 and $\mathrm{B}$ is the second plotted point. $R_{1}$ and $R_{2}$ are ranges of echoes and $\Delta Z$ is the difference between two bearings. $A B$ is the distance run on the relative track. Hence, $D$ (OP) is the estimated distance of the C.P.A.

Then,

$$
D=\frac{R_{1} R_{2} \sin \Delta Z}{\sqrt{ }\left(R_{1}{ }^{2}+R_{2}{ }^{2}-2 R_{1} R_{2} \cos \Delta Z\right)}
$$

In case of the vessels crossing at a fine-angle, the change of bearing $\Delta Z$ is small, and

$$
D \bumpeq 0.0175 \Delta Z^{\circ} \frac{R_{1} R_{2}}{R_{1}-R_{2}}
$$

From the above formula, the distance of the C.P.A. is proportional to the bearing change and the square of the mean range, and approximately inversely proportional to range change. 
Now, if it is expected that the estimated distance of C.P.A. is 3 miles in fog, the relation between the bearing and the range change should be as shown in Fig. 2. When the first range is 10 miles and the range change is 2 miles, change of bearing must be $4: 6$ or more. When the first range is ro miles and bearing change is $3^{\circ}$, the range change must be 1.4 miles or less.

If the expected distance of C.P.A. is I mile, about $\frac{1}{3}$ of bearing change of the above value will do. The scale of degrees on the right-hand side in Fig. 2 shows an approximate bearing change in this case.

Now, according to Topley, the radius of the error circle of a plotted point due to range and bearing errors is $0.03 R$, where $R$ is the mean range. Since the error in estimated distance of C.P.A. is $\Delta D$ (PQ) in Fig. 1 , then

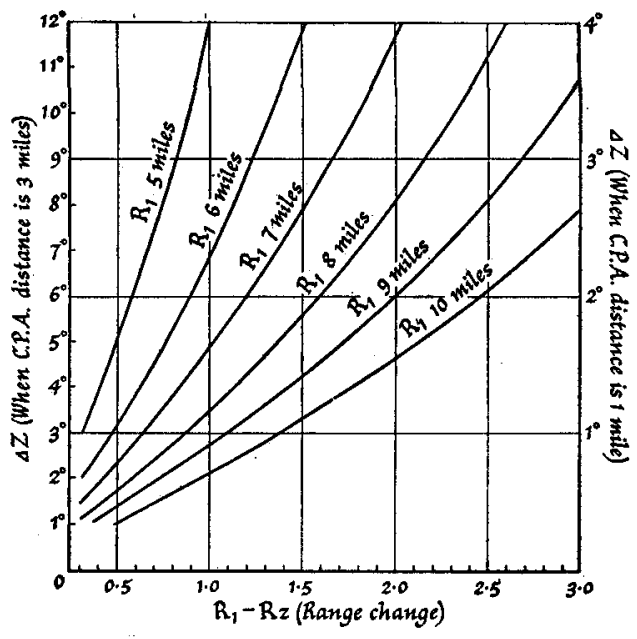

Fig. 2

$$
\Delta D=\frac{0.03 R R_{1} \cos A}{\sqrt{ }\left(R_{1}{ }^{2}+R_{2}{ }^{2}-2 R_{1} R_{2} \cos \Delta Z\right)}
$$

And since $R \cos A \bumpeq R_{2}$, and $\Delta Z$ is small,

$$
\Delta D \bumpeq 0.03 \frac{R_{1} R_{2}}{R_{1}-R_{2}}
$$

From the above formula, an error in C.P.A. is also approximately proportional to the square of the mean range and inversely proportional to range change, as is distance of C.P.A. Rate of error of distance of C.P.A. seems to be more convenient in practice than an absolute value of the error.

Therefore, $(3) \div(1)$ or $(4) \div(2)$

$$
\frac{\Delta D}{D} \bumpeq 1 \cdot 7^{2} \frac{\mathrm{I}}{\Delta Z^{\circ}}
$$

This formula is very simple, but will give fairly good results. From this, rate of error is inversely proportional to bearing change, not being concerned with others. If the plotting interval is doubled (bearing change $\times 2$ ), the error rate would be halved. In practice, change of rate of bearing will become important, since the plotting interval will be limited to some extent.

If the bearing change is the same, the rate will be the same. Therefore, it cannot be said that the rate of error is always small when the range is small, or that the range change is large, as it depends upon bearing change only.

In Fig. 3 drawn from formula (5), the error rate is 60 per cent when the bearing change is $3^{\circ}, 35$ per cent at $5^{\circ}$ change and less than 20 per cent at more than $9^{\circ}$ change. If a bearing change is $1^{\circ}$, then $\Delta D=1 \cdot 7^{2} \mathrm{D}$. It is not clear on which side the ship will pass, as the error is larger than the estimated distance of C.P.A. 


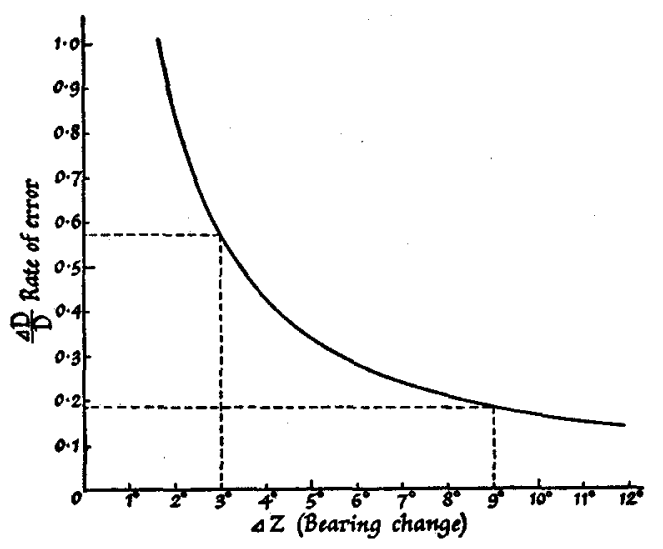

Fig. 3

It can be said from the above that it is necessary to have at least $3^{\circ}$ bearing change between two observations, so that a maximum error rate is 60 per cent. Further, it is desirable that an estimated distance of C.P.A. is 3 miles in fog, when the maximum error will be I.8 miles and the remaining distance $\mathrm{I} \cdot 2$ miles.

If an estimated distance of C.P.A. is I mile, maximum error is 0.6 mile and the remaining distance is only 0.4 mile, which will be considered dangerous in fog.

\section{THE UNDER-THIRTY PRIZE}

The Council of the Institute has decided to offer an annual prize, to be known as the Under-thirty Prize, for the most notable paper to be submitted for publication by anyone under thirty years of age. The prize will be twenty-five guineas.

Papers should not exceed 3500 words and should reach the Executive Secretary by $3 \circ$ June in any year. Four copies should be submitted. The author's name should not appear on the paper but a covering letter should state the author's intention of entering the paper for the competition.

All papers submitted will normally be treated as available for publication in the Journal. 